\title{
Association between serum non-high- density lipoprotein cholesterol and cognitive impairment in patients with acute ischemic stroke
}

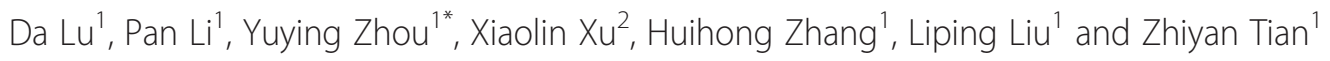

\begin{abstract}
Background: Non-high density lipoprotein cholesterol (HDL-C) could be a good predictor of vascular disease outcomes. To evaluate the association between serum non-HDL-C and cognitive impairment in patients with acute ischemic stroke.

Methods: A total of 725 hospitalized patients with acute ischemic stroke were enrolled. They received conventional treatment. Cognitive function was assessed on the 3rd day after admission using mini-mental state examination (MMSE), Montreal Cognitive Assessment (MoCA), Activity of Daily Living Scale (ADL), and Neuropsychiatric Inventory (NPI, and Hamilton depression rating scale 21-item (HAMD-21). Lipid profile and biochemical markers were measured, and non-HDL-C was calculated.

Results: Compared with patients with normal non-HDL-C, those with high non-HDL-C showed lower MMSE ( $23.1 \pm 4.9$ vs. $26.0 \pm 4.6, P<0.001)$ and MoCA ( $20.4 \pm 6.4$ vs. $22.2 \pm 5.3 P=0.01)$ scores, higher NPI (6.2 \pm 1.2 vs. $3.3 \pm 1.5, P<0.001)$ and HADM-21 (6.0 \pm 2.2 vs. $4.5 \pm 1.9, P<0.001)$ scores, and higher homocysteine $(16.0 \pm 3.8$ vs. $14.3 \pm 2.0 \mathrm{mmol} / \mathrm{L}, P=0.044)$, fasting blood glucose $(6.4 \pm 2.7$ vs. $6.1 \pm 2.1 \mathrm{mmol} / \mathrm{L}, P=0.041)$, and $\mathrm{HbA} 1 \mathrm{c}$ $(6.80 \pm 1.32 \%$ vs. $6.52 \pm 1.17 \%, P=0.013)$ levels. MMSE $(r=-0.526, P<0.001), \operatorname{MoCA}(r=-0.216, P<0.001)$, and NPI $(r=0.403, P<0.001)$ scores were correlated with non-HDL-C levels. High non-HDL-C levels were an independent risk factor for cognitive disorders after acute ischemic stroke $(P=0.034$, odds ratio $=3.115,95 \%$ confidence interval: 1.088-8.917).

Conclusions: High serum non-HDL-C levels, age, education, homocysteine levels, and HAMD score were independent risk factors of cognitive impairment in patients with acute ischemic stroke. The risk of cognitive disorders after acute ischemic stroke increased with increasing non-HDL-C levels. This parameter is easy to assess in the clinical setting.
\end{abstract}

Keywords: Dyslipidemia, Non-high-density lipoprotein cholesterol, Ischemic stroke, Cognitive disorders

Abbreviations: ADL, Activity of Daily Living Scale; BMI, Body Mass Index; HADM-21, Hamilton Depression Rating Scale 21-Item; HbA1c, Glycated Hemoglobin; HCY, Homocysteine; HDL-C, High-Density Lipoprotein Cholesterol; LACl, Lacunar Circulation Infarction; LDL-C, Low-Density Lipoprotein Cholesterol; MMSE, Mini-Mental State Examination; MoCA, Montreal Cognitive Assessment; non-HDL-C, Non-High-Density Lipoprotein Cholesterol; NPI, Neuropsychiatric Inventory; PACl, Partial Anterior Circulation Infarction; POCl, Posterior Circulation Infarction; S-CRP, Serum High-Sensitivity C-Reactive Protein; TACI, Total Anterior Circulation Infarction; TC, Total Cholesterol; TG, Triglycerides

\footnotetext{
* Correspondence: qiying789@sina.cn

'Department of Neurology, Sixteenth wards, Tianjin Huanhu Hospital, Tianjin 300060, China

Full list of author information is available at the end of the article
} International License (http://creativecommons.org/licenses/by/4.0/), which permits unrestricted use, distribution, and reproduction in any medium, provided you give appropriate credit to the original author(s) and the source, provide a link to the Creative Commons license, and indicate if changes were made. The Creative Commons Public Domain Dedication waiver (http://creativecommons.org/publicdomain/zero/1.0/) applies to the data made available in this article, unless otherwise stated. 


\section{Background}

Acute ischemic stroke is an episode of neurological dysfunction caused by cerebral ischemia persisting for $>24 \mathrm{~h}$ or until death [1], and represent $80-87 \%$ of all strokes [2, 3]. In high income countries, the incidence of stroke is 217 per 100,000 person-years and the prevalence is 715 per 100,000 people, compared with low income countries where the incidence is 281 per 100,000 person-years and the prevalence is 393 per 100,000 people. Likely risk factors include history of transient ischemic attacks, cardiovascular diseases (hypertension, myocardial infarction), smoking, metabolic syndrome, heavy alcohol consumption, type 2 diabetes mellitus, high cholesterol levels, carotid artery stenosis, genetic factors, and biochemical factors [2, 3].

Vascular cognitive impairment (VCI) is one of the most common non-somatic manifestations in patients with acute ischemic stroke [4]. Dyslipidemia is not only an independent and maybe the most important risk factor for ischemic stroke, which tends to cause hidden, progressive, and somatic organic damage, but also affects the cognitive function of patients with ischemic stroke through accelerating systemic atherosclerosis, and it is believed to be a risk factor inducing cognitive disorders, even dementia [5].

Previous guidelines for blood lipid treatment preferred low-density lipoprotein cholesterol (LDL-C) as the primary target of lipid-lowering therapy [6]. However, some recent studies and epidemiological investigations indicated that serum non-high-density lipoprotein cholesterol (HDL-C) levels were superior to LDL-C in terms of predicting the risk of cardiovascular diseases. The National lipid association recommendations for patientcentered management of dyslipidemia issued by the American National Lipid Association (NLA) in 2015 proposes that non-HDL-C is preferable to LDL-C as the primary target of interventions [7], since non-HDL-C includes all lipoprotein particles other than the HDLs, therefore including the triglyceride-rich lipoproteins [chylomicrons, very-low-density lipoproteins (VLDL), and their remnants], which are now known to participate in atherosclerosis [7]. Nevertheless, the exact relationship between non-HDL-C and cognitive impairment after stroke is still poorly understood.

Therefore, this study aimed to investigate the association between serum non-HDL-C levels and cognitive function after stroke, as well as the value of non-HDL-C levels in assessing the risk of cognitive disorders after acute ischemic stroke.

\section{Methods}

\section{Study design}

This was a retrospective case-control study of patients with acute ischemic stroke hospitalized and registered at the Department of Neurology of the Tianjin Huanhu
Hospital between January 2010 and December 2015. The study was approved by the ethical committee of the Tianjin Huanhu Hospital. The need for individual consent was waived by the committee because of the retrospective nature of the study.

\section{Patients}

Inclusion criteria were: 1) newly diagnosed with ischemic stroke according to the China cerebral vascular disease guidelines [8] based on computed tomography (CT) and/ or magnetic resonance imaging (MRI); 2) onset time $\leq 7$ days; and 3 ) aged $>18$ years old. Exclusion criteria were: 1) disturbance of consciousness, severe aphasia, or hemiplegia, and unable to complete neuropsychological testing; 2) Alzheimer's disease (AD), depression, dementia with Lewy bodies (DLB), frontotemporal dementia (FTD), or dementia caused by other diseases such as malignant tumors, intracranial infection, neurodegenerative diseases, craniocerebral trauma, etc.; 3) heart, lung, liver, kidney, or endocrine system diseases, or connective tissue diseases, blood disease, or malnutrition; 4) history of mental diseases or behavior disorders; 5) history of ischemic stroke with cognitive disorders based on the medical files or inquiry to the family at admission; or 6) history of nootropics or antipsychotic drugs within 4 weeks.

\section{Data collection}

Patients' gender, age, height, body mass index (BMI), level of education, family history of dementia, presence of vascular risk factors such as hypertension, diabetes, hyperlipidemia, heart diseases (atrial fibrillation, myocardial infarction, angina pectoris, asystole, etc.), history of stroke and/or transient ischemic attack (TIA), smoking history, and drinking history were recorded in details at admission.

\section{Grouping}

The patients were divided into three subgroups according to the Oxfordshire Community Stroke Project (OCSP) classification [9]: 1) total/partial anterior circulation infarction (TACI/PACI) group; 2) posterior circulation infarction (POCI) group; and 3) lacunar circulation infarction (LACI) group. TACI manifests as a triad, i.e. manifestations of complete middle cerebral artery syndrome: 1 ) cerebral and high nervous disorders (disturbance of consciousness, aphasia, acalculia, spatial disorientation, etc.); 2) homonymous hemianopia or conjugate eye deviation; and 3) motor and/or sensory disturbance at three contralateral parts (face, upper limbs, and lower limbs). PACI manifests as two out of three manifestations. POCI manifests as various degrees of vertebro-basilar artery syndromes: 1) ipsilateral cerebral palsy and contralateral sensory disorders; 2) bilateral sensory and motor disorders; 3) collaborative movement disorders of eyes and cerebellar dysfunction, 
which are unequal brain-stem and cerebellar infarctions caused by obstruction of vertebro-basilar artery and branches. LACI manifests as lacunar syndrome.

\section{Biochemistry}

Overnight fasting blood samples were routinely drawn. Fasting blood glucose, total cholesterol (TC), triglycerides (TG), HDL-C, and LDL-C were measured using an ADVIA-2400 automatic biochemical analyzer (Siemens, Erlangen, Germany) and the triglyceride test kit (GPOPAP, Shanghai Huachen, Shanghai, China). A BNP II protein analyzer (Siemens, Erlangen, Germany) was used to test serum high-sensitivity CRP (hs-CRP) (C-reactive protein kit, Siemens Healthcare Diagnostics Products $\mathrm{GmbH}$, Erlangen, Germany), glycated hemoglobin (HbA1c) (HBIC HbA1c kit, Siemens Healthcare Diagnostics Products $\mathrm{GmbH}$, Erlangen, Germany), and homocysteine (Hcy) (Hcy kit, Siemens Healthcare Diagnostics Products $\mathrm{GmbH}$, Erlangen, Germany).

Non-HDL-C was calculated by subtracting HDL-C levels from TC. Non-HDL-C levels were classified into two grades according to the criteria recommended by the Patient-centered management of dyslipidemia developed by the NLA in 2015 [7]: normal group (non-HDL-C $<3.4 \mathrm{mmol} / \mathrm{l}$ ) and high group (non-HDL-C $\geq 3.4 \mathrm{mmol} / \mathrm{l}$ ). The high group can be further divided into higher than normal $(3.4 \mathrm{mmol} / \mathrm{l} \leq$ non-HDL-C $<4.2 \mathrm{mmol} / \mathrm{l})$, critical high $(4.2 \mathrm{mmol} / \mathrm{l} \leq$ non-HDL-C $<5.0 \mathrm{mmol} / \mathrm{l})$, and very high (non-HDL-C $\geq 5.0 \mathrm{mmol} / \mathrm{l}$ ).

\section{Neurological assessment}

After admission, patients were routinely given antiplatelet, anticoagulation, expansion, and other conventional treatments for stroke [3]. On the 3rd day after admission, their neuropsychological scales were routinely assessed by trained and skilled neurologists. Cognitive function was screened using the mini-mental state examination (MMSE) [10] and the Montreal Cognitive Assessment (MoCA) [11] scales. The total score of MMSE is 30 points, of which $<17$ points, $<20$ points, and $<24$ points in illiterate patients, patients with education level of primary school, and patients with education level of secondary school or above, respectively, are considered as cognitive disorders. The total score of MoCA is 30 points, where a score $<26$ points is considered as cognitive disorders, using a score +1 to correct for education level for patients with $\leq 12$ years of education. MoCA presents a higher sensitivity in screening mild cognitive disorders, while MMSE is more effective and convenient for dementia patients with multiple-domain impairment. In this study, results of the above two scales were comprehensively considered since they were only used as quantitative indices of $\operatorname{cog}$ nitive level variation with the change of non-HDL-C.
Neuropsychological behavior was assessed using the neuropsychiatric inventory (NPI) [12] to determine the frequency and severity of behavioral disorders as well as distress. The NPI included 12 items and a total score of 144 points, where a higher score refers to more severe psychological and behavioral disorders.

The activities of daily living (ADL) were assessed using the ADL scale [13], which includes 20 items: 11 items for basic ADL (BADL) and 9 items of instrumental ADL (IADL), for a total score of 80 points, where a higher score refers to a more severe impairment.

The emotional state was assessed using the Hamilton Rating Scale 21-item HAMD-21 [14], where a score $\geq 7$, $\geq 17$, and $\geq 24$ points refers to mild, moderate, and severe depression, respectively.

\section{Statistical analysis}

Statistical analysis was performed using SPSS 19.0 (IBM, Armonk, NY, USA). Normally-distributed continuous data are presented as mean \pm standard deviation and were analyzed using the independent-samples $t$-test or ANOVA with the LSD post hoc test, as appropriate. Categorical data are expressed as frequency and were analyzed using the chi-square test. Correlations were assessed using the Pearson correlation test. Logistic regression (stepwise) was performed to determine the factors independently associated with higher cognitive impairment. Two-sided $P$-values $<0.05$ were considered statistically significant.

\section{Results}

\section{Characteristics of the patients}

A total of 725 patients with acute cerebral infarction were hospitalized at the Department of Neurology of the Tianjin Huanhu Hospital between October 2010 and March 2015. They were divided into two groups according to the non-HDL-C levels: the normal group included 253 patients (192 men and 61 women, aged $54-85$ years, mean age of $63.1 \pm 11.9$ years) and the high group included 472 patients (336 men and 136 women, aged $52-81$ years, mean age of $62.2 \pm 10.8$ years). Furthermore, each group was further divided into three subgroups according to the OCSP classification: TACI/PACI, POCI, and LACI.

Gender, age, years of education, as well as hypertension, diabetes, coronary atherosclerotic heart disease, smoking history, drinking history, family history of dementia, and stroke history were similar between the two groups $(P>0.05)$ (Table 1$)$, but BMI in the normal nonHDL-C group was significantly lower than in the high non-HDL-C group $(P=0.009)$ (Table 1$)$. TC, TG, HDL$\mathrm{C}$, LDL-C, HCY, fasting blood glucose, HbA1c, and other indicators were statistically different between the two groups $(P<0.05)$ (Table 1$)$, except for hsCRP $(P>$ $0.05)$. There was no significant difference in OCSP subtypes between the two groups $(P>0.05)$. 
Table 1 Socio-demographic and clinical characteristics of the patients

\begin{tabular}{|c|c|c|c|}
\hline Variables & Normal non-HDL-C $(n=253)$ & High non-HDL-C $(n=472)$ & $P$ \\
\hline \multicolumn{4}{|l|}{ Gender, n (\%) } \\
\hline M & $192(75.9)$ & $336(71.2)$ & \multirow[t]{2}{*}{0.175} \\
\hline $\mathrm{F}$ & $61(24.1)$ & $136(28.8)$ & \\
\hline Age (years) & $63.1 \pm 11.9$ & $62.2 \pm 10.8$ & 0.289 \\
\hline BMI $\left(\mathrm{kg} / \mathrm{m}^{2}\right)$ & $24.6 \pm 3.4$ & $25.3 \pm 3.3$ & 0.009 \\
\hline Years of education (years) & $9.2 \pm 4.3$ & $9.1 \pm 3.9$ & 0.788 \\
\hline Hypertension, n (\%) & $167(66.0)$ & $317(67.2)$ & 0.753 \\
\hline Diabetes, n (\%) & $58(22.9)$ & $101(21.4)$ & 0.636 \\
\hline Coronary atherosclerotic heart disease, n (\%) & $43(17.0)$ & 88 (18.6) & 0.583 \\
\hline Smoking history, n (\%) & $90(35.6)$ & $168(35.6)$ & 0.996 \\
\hline Drinking history, n (\%) & $67(26.5)$ & $122(25.9)$ & 0.853 \\
\hline Family history of dementia, n (\%) & $10(4.0)$ & $19(4.0)$ & 0.962 \\
\hline Stroke history, n (\%) & $94(37.2)$ & $151(32.0)$ & 0.161 \\
\hline Non-HDL-C (mmol/l) & $2.77 \pm 0.46$ & $4.40 \pm 0.86$ & $<0.001$ \\
\hline $\mathrm{TC}(\mathrm{mmol} / \mathrm{l})$ & $3.86 \pm 0.52$ & $5.54 \pm 0.88$ & $<0.001$ \\
\hline TG $(\mathrm{mmol} / \mathrm{l})$ & $1.27 \pm 0.56$ & $1.95 \pm 0.31$ & $<0.001$ \\
\hline $\mathrm{HDL}-\mathrm{C}(\mathrm{mmol} / \mathrm{l})$ & $1.08 \pm 0.26$ & $1.13 \pm 0.25$ & 0.027 \\
\hline LDL-C (mmol/l) & $2.16 \pm 0.46$ & $3.39 \pm 0.80$ & $<0.001$ \\
\hline $\mathrm{HCY}(\mathrm{mmol} / \mathrm{l})$ & $14.28 \pm 2.01$ & $16.02 \pm 3.80$ & 0.044 \\
\hline hsCRP (mg/l) & $4.35 \pm 0.78$ & $4.47 \pm 1.97$ & 0.895 \\
\hline Fasting glucose (mmol/l) & $6.05 \pm 2.06$ & $6.44 \pm 2.66$ & 0.041 \\
\hline $\mathrm{HbA1c}(\%)$ & $6.52 \pm 1.17$ & $6.80 \pm 1.32$ & 0.013 \\
\hline TACI/PACl, n (\%) & $167(66.0)$ & $309(65.5)$ & 0.264 \\
\hline $\mathrm{POCl}, \mathrm{n}(\%)$ & $67(26.5)$ & $111(23.5)$ & \\
\hline LACI, n (\%) & $19(7.5)$ & $52(11.0)$ & \\
\hline
\end{tabular}

$B M I$ body mass index, non- $H D L-C$ non-high-density lipoprotein cholesterol, $T C$ total cholesterol, $T G$ triglycerides, $H D L-C$ high-density lipoprotein cholesterol, $L D L-C$ low-density lipoprotein cholesterol, $H C Y$ homocysteine, $h S C R P$ high-sensitivity C-reactive protein, $H b A 1 c$ glycated hemoglobin, $T A C I$ total anterior circulation infarction, $\mathrm{PACl}$ partial anterior circulation infarction, $\mathrm{POCl}$ posterior circulation infarction, $\mathrm{LACl}$ lacunar circulation infarction

\section{Impact of non-HDL-C levels on cognitive impairment}

The MMSE and MOCA scores in the high non-HDL-C group were significantly lower than in the normal nonHDL-C group (MMSE: $P<0.001$; MoCA: $P=0.01$ ). Assessments of neuropsychological behavior and emotion showed that the NPI and HADM-21 scores in the high non-HDL-C group were higher than in the normal nonHDL-C group $(P<0.001)$. Meanwhile, the ADL scale scores were similar between the two groups $(P>0.05)$ (Table 2).

\section{Association of non-HDL-C levels and neurological scores} The high non-HDL-C group was further divided into the higher than normal non-HDL-C (208 cases), critical high non-HDL-C (183 cases), and very high nonHDL-C (81 cases). The NIHSS was significantly higher in patients with critical high non-HDL-C levels compared with patients with higher than normal levels $(P<0.05)$ (Table 3$)$.

\section{Correlations of non-HDL-C level with various indices among patients with high non-HDL-C levels}

Pearson correlation analyses revealed that MMSE (weak correlation, $r=-0.237, P<0.001$ ) and MoCA (weak correlation, $r=-0.194, P<0.001)$ scores were negatively correlated with non-HDL-C level in patients with acute

Table 2 Comparison of neuropsychological scale between the two groups

\begin{tabular}{lccl}
\hline & $\begin{array}{l}\text { Normal non-HDL-C } \\
(n=253)\end{array}$ & $\begin{array}{l}\text { High non-HDL-C } \\
(n=472)\end{array}$ & $P$ \\
\hline MMSE & $26.0 \pm 4.6$ & $23.1 \pm 4.9$ & $<0.001$ \\
MoCA & $22.2 \pm 5.3$ & $20.4 \pm 6.4$ & 0.010 \\
NPI & $3.3 \pm 1.5$ & $6.2 \pm 1.2$ & $<0.001$ \\
HADM-21 & $4.5 \pm 1.9$ & $6.0 \pm 2.2$ & $<0.001$ \\
ADL & $25.7 \pm 8.0$ & $28.2 \pm 4.1$ & 0.230 \\
\hline
\end{tabular}

MMSE mini-mental state examination, MoCA Montreal Cognitive Assessment, NPI Neuropsychiatric Inventory, ADL Activity of Daily Living Scale, HADM-21 Hamilton depression rating scale 21-item 
Table 3 Comparison of the non-HDL-C differences according to neurological scores

\begin{tabular}{lcccc}
\hline & $\begin{array}{l}\text { Higher than } \\
\text { normal non- } \\
\text { HDL-C levels } \\
\text { (208 cases) }\end{array}$ & $\begin{array}{l}\text { Critical high } \\
\text { non-HDL-C } \\
\text { levels } \\
(183 \text { cases })\end{array}$ & $\begin{array}{l}\text { Very high } \\
\text { non-HDL-C } \\
\text { levels } \\
\text { (81 cases) }\end{array}$ & $P$ \\
\hline NIHSS & $4.0 \pm 1.3$ & $3.6 \pm 1.1^{\mathrm{a}}$ & $4.8 \pm 1.8$ & 0.043 \\
NPI & $3.9 \pm 1.2$ & $4.1 \pm 1.2$ & $4.9 \pm 1.5^{\text {ab }}$ & 0.021 \\
BI & $84.1 \pm 14.6$ & $84.8 \pm 14.4$ & $82.9 \pm 16.1$ & 0.217 \\
MMSE & $24.9 \pm 4.6$ & $24.6 \pm 5.1$ & $22.6 \pm 6.0^{\mathrm{ab}}$ & $<0.001$ \\
MoCA & $20.5 \pm 5.0$ & $20.6 \pm 5.9$ & $18.3 \pm 4.3^{\mathrm{ab}}$ & 0.002 \\
ADL & $26.3 \pm 8.2$ & $26.5 \pm 8.4$ & $29.2 \pm 10.3$ & 0.450 \\
HAMD & $4.8 \pm 1.3$ & $4.7 \pm 1.0$ & $4.2 \pm 2.7$ & 0.634 \\
\hline
\end{tabular}

NIHSS NIH stroke score, MMSE mini-mental state examination, MoCA Montreal Cognitive Assessment, NPI, Neuropsychiatric Inventory, ADL Activity of Daily Living Scalem, HADM-21 Hamilton depression rating scale 21-item ${ }^{a}$ ANOVA and LSD post hoc test: $P<0.05$ vs. the higher than normal non-HDL-C group

${ }^{\mathrm{b}}$ ANOVA and LSD post hoc test: $P<0.05$ vs. the critical high non-HDL-C group

ischemic stroke. In order to correct the impacts of gender, age, education level, disease history, and other confounding factors, we further performed partial correlation analyses, which showed that MMSE (moderate correlation, $r=-0.526, P<0.001)$ and $\mathrm{MoCA}$ (weak correlation, $r=-0.216, P<0.001$ ) scores were negatively correlated with non-HDL-C levels, while NPI scores (weak correlation, $r=0.301, \quad P<0.001$ ) were positively correlated with non-HDL-C levels (Table 4).

\section{Effects of stroke site on cognitive function}

Assessment of cognitive function showed that MMSE and MoCA scores were significantly different among different subtypes of cerebral infarction in the two groups $(P<0.05)$, of which the score was the lowest in the TACI/PACI group, followed by the POCI and LACI groups. In addition, among different cerebral infarction subtypes, the MMSE and MoCA scores were significantly lower in the high level group compared with the normal level group $(P<0.001)$ (Table 5).

Table 4 Correlation of non-HDL-C levels with neurological scores

\begin{tabular}{lllllll}
\hline Items & \multicolumn{2}{c}{ Pearson correlation analysis } & & \multicolumn{2}{c}{ Partial correlation analysis } \\
\cline { 2 - 3 } \cline { 6 - 7 } & $r$ & & $P$ & & \multicolumn{2}{c}{$P$} \\
\hline MMSE & -0.237 & $<0.001$ & & -0.526 & $<0.001$ \\
NPI & 0.059 & 0.2 & & 0.301 & $<0.001$ \\
MoCA & -0.194 & $<0.001$ & & -0.216 & $<0.001$ \\
HAMD-21 & 0.028 & 0.494 & & 0.046 & 0.316 \\
ADL & 0.036 & 0.388 & & 0.047 & 0.311 \\
\hline
\end{tabular}

The partial correlation analysis is corrected for the impact of gender, age, education level, and disease history

MMSE mini-mental state examination, MoCA Montreal Cognitive Assessment, NPI Neuropsychiatric Inventory, ADL Activity of Daily Living Scale, HADM-21 Hamilton depression rating scale 21-item
Table 5 Comparison of MMSE and MoCA scores of patients with different cerebral infarctions between the two groups

\begin{tabular}{llll}
\hline & Normal level $(n=253)$ & High level $(n=472)$ & $P$ \\
\hline MMSE & & & \\
TACI/PACl & $28.49 \pm 1.19$ & $21.94 \pm 5.13$ & $<0.001$ \\
POCl & $29.01 \pm 0.83$ & $22.47 \pm 4.22$ & $<0.001$ \\
LACl & $29.50 \pm 0.51$ & $23.98 \pm 4.04$ & $<0.001$ \\
$P$ & $<0.001$ & 0.019 & \\
MoCA & & & $<0.001$ \\
TACI/PACl & $25.10 \pm 2.97$ & $17.29 \pm 6.50$ & $<0.001$ \\
POCI & $26.05 \pm 2.68$ & $17.95 \pm 5.68$ & $<0.001$ \\
LACI & $27.35 \pm 1.93$ & $19.62 \pm 5.72$ & \\
$P$ & 0.003 & 0.045 & \\
\hline
\end{tabular}

MMSE mini-mental state examination, MoCA Montreal Cognitive Assessment, TACI/PACI total/partial anterior circulation infarction, $P O C /$ posterior circulation infarction, $L A C l$ lacunar circulation infarction

Risk analysis of non-HDL-C levels to cognitive impairment after ischemic stroke

Univariable analyses revealed that non-HDL-C levels, education level, age, history of diabetes, history of stroke, family history of dementia, HAMD score, and HCY level $(P<0.05)$ were associated with cognitive disorders after acute ischemic stroke (Table 6). These factors were included in the multivariable analysis and the results showed that high non-HDL-C levels, history of stroke, low education level, age, HAMD score, and HCY levels were independently associated with cognitive disorders after ischemic stroke (all $P<0.05$ ).

\section{Discussion}

Non-HDL-C could be a good predictor of vascular disease outcomes. Therefore, this study aimed to evaluate the association between serum non-HDL-C and cognitive impairment in patients with acute ischemic stroke. Results showed that compared with normal non-HDL-C, patients with high non-HDL-C showed lower MMSE and MoCA scores, higher NPI and HADM-21 scores, and higher $\mathrm{HCY}$, fasting blood glucose, and HbA1c levels. MMSE, MoCA, and NPI scores were correlated with non-HDL-C levels. High non-HDL-C levels were an independent risk factor of cognitive disorders after acute ischemic stroke.

Non-HDL-C was initially used to predict the risk of cardiovascular diseases and is a strong predictor for allcause mortality and cardiovascular disease mortality, and is significantly superior to LDL-C levels [15]. The Bypass Angioplasty Revascularization Investigation (BARI) study assessed the efficacy of secondary prevention in 1514 patients with multiple coronary diseases, and their 5-year follow-up revealed that non-HDL-C levels were an independent predictor for non-fetal myocardial infarction, while LDL-C levels failed to have significant predictive 
Table 6 Univariable and multivariable analyses of factors associated with cognitive disorders after acute ischemic stroke

\begin{tabular}{|c|c|c|c|c|c|c|}
\hline \multirow[b]{2}{*}{ Variables } & \multicolumn{3}{|c|}{ Univariable } & \multicolumn{3}{|c|}{ Multivariable } \\
\hline & $P$ & OR & $95 \% \mathrm{Cl}$ & $P$ & OR & $95 \% \mathrm{Cl}$ \\
\hline Gender, male & 0.239 & 0.729 & $0.431-1.234$ & & & \\
\hline Age & 0.001 & 1.043 & $1.018-1.068$ & 0.001 & 1.039 & $1.016-1.064$ \\
\hline BMI & 0.623 & 0.982 & $0.913-1.056$ & & & \\
\hline Years of education & $<0.001$ & 0.817 & $0.772-0.866$ & $<0.001$ & 0.808 & $0.757-0.861$ \\
\hline Hypertension & 0.324 & 1.207 & $0.831-1.755$ & & & \\
\hline Diabetes & $<0.001$ & 1.276 & $1.175-1.385$ & 0.341 & 1.400 & $0.700-2.797$ \\
\hline Coronary heart disease & 0.388 & 1.380 & $0.664-2.870$ & & & \\
\hline History of smoking & 0.367 & 1.226 & $0.788-1.907$ & & & \\
\hline History of drinking & 0.524 & 1.221 & $0.661-2.254$ & & & \\
\hline Family history of dementia & 0.001 & 1.219 & $1.082-1.374$ & 0.055 & 0.242 & $0.057-1.031$ \\
\hline History of stroke & 0.021 & 1.742 & $1.087-2.791$ & 0.025 & 1.723 & $1.071-2.771$ \\
\hline High TC & 0.629 & 0.791 & $0.306-2.044$ & & & \\
\hline High TG & 0.674 & 1.078 & $0.760-1.529$ & & & \\
\hline Low HDL-C & 0.088 & 0.650 & $0.396-1.066$ & & & \\
\hline High LDL-C & 0.900 & 1.025 & $0.694-1.514$ & & & \\
\hline High Non-HDL-C & 0.034 & 3.115 & $1.088-8.917$ & $<0.001$ & 3.115 & $1.088-8.917$ \\
\hline Normal level & 0.170 & 1.691 & $0.799-3.579$ & 0.088 & 1.691 & $0.799-3.579$ \\
\hline Higher than normal & 0.046 & 2.710 & $1.017-7.221$ & 0.052 & 2.710 & $1.017-7.221$ \\
\hline Critical high level & 0.026 & 5.877 & $1.231-28.065$ & 0.040 & 5.877 & $1.231-28.065$ \\
\hline Very high level & 0.013 & 7.006 & $1.502-32.675$ & 0.932 & 7.006 & $1.502-32.675$ \\
\hline High Hcy & $<0.001$ & 1.060 & $1.032-1.088$ & $<0.001$ & 1.057 & $1.031-1.084$ \\
\hline hsCRP & 0.515 & 0.990 & $0.960-1.021$ & & & \\
\hline Fasting hyperglycemia [n (\%)] & 0.285 & 1.084 & $0.935-1.256$ & & & \\
\hline High HbA1c & 0.960 & 1.002 & $0.939-1.068$ & & & \\
\hline NPI & 0.218 & 1.039 & $0.978-1.104$ & & & \\
\hline HAMD-21 & 0.001 & 1.224 & $1.089-1.375$ & 0.001 & 1.284 & $1.177-1.401$ \\
\hline$A D L$ & 0.141 & 1.012 & $0.996-1.029$ & & & \\
\hline
\end{tabular}

$B M I$ body mass index, Non-HDL-C non-high-density lipoprotein cholesterol, $T C$ total cholesterol, $T G$ triglycerides, $H D L-C$ high-density lipoprotein cholesterol, $L D L-C$ low-density lipoprotein cholesterol, $H C Y$ homocysteine, $h S C R P$ serum high-sensitivity C-reactive protein, $H b A 1 c$ glycated hemoglobin, MMSE mini-mental state examination, MoCA Montreal Cognitive Assessment, NPI Neuropsychiatric Inventory, ADL Activity of Daily Living Scale, HADM-21 Hamilton depression rating scale 21-item

effect on the primary endpoint or mortality [16]. In another clinical longitudinal study with a follow-up $>19$ years in 2406 men and 2058 women, the risk of coronary disease in men with non-HDL-C levels $>220 \mathrm{mg} / \mathrm{dl}$ was 2.14 times that of men with non-HDL-C levels $<160 \mathrm{mg} / \mathrm{dl}$, while that in people with LDL-C levels $>190 \mathrm{mg} / \mathrm{dl}$ was 1.77 times that of people with LDL-C levels $<130 \mathrm{mg} / \mathrm{dl}$ [15]. Recent studies proposed that serum non-HDL-C levels were closely associated with cerebral vascular diseases. Indeed, $\mathrm{Wu}$ et al. $[17,18]$ conducted a follow-up study of 95,916 18-98 year-old patients without stroke or myocardial infarction in Tangshan (Hebei, China) and found that serum non-HDL-C levels were an independent risk factor of ischemic stroke, and that high serum
non-HDL-C levels were associated with an increase of $>50 \%$ of the risk of ischemic stroke in the healthy population, which was better than the association between LDL$\mathrm{C}$ and the same risk. In their study, $13.0 \%$ of the patients had asymptomatic intracranial arterial stenosis (ICAS) $[17,18]$. In addition, they found that the elevation extent of serum non-HDL-C levels was positively correlated with ICAS incidence, and was an independent risk factor for it $(\mathrm{OR}=1.15)$ [19]. Patients with mild cognitive impairment (MCI) caused by intracranial stenosis are prone to deteriorate and progress to dementia, and its mechanism might be because atherosclerosis cause vascular distortion and enwinding, but also might induce mechanical occlusion of intracranial vessels. In addition, an important 
atherosclerotic stenosis might indicate systemic atherosclerosis and extensive microvascular diseases, damage of microcirculation, increased resistance of small vessels and decreased vascular reactivity, ultimately leading to cerebral hypoperfusion. The cumulative effect of these interdependent hemodynamic disorders might play a key role in accelerating the onset and progression of cognitive disorders [20]. Some studies also found that serum nonHDL-C levels were significantly increased in patients with $\mathrm{MCI}$ compared with people with normal cognitive function, and the cognitive score was negatively correlated with the non-HDL-C levels $(r=-0.761)$ [21]. This crosssectional analysis first discovered that the non-HDL-C levels in $65.1 \%$ of the patients with acute ischemic stroke was significantly higher than the normal value, and that it was positively correlated with the prevalence and damage extent of cognitive impairment after acute ischemic stroke. Thus, serum non-HDL-C level might have high value in predicting the disease since it was an independent risk factor of vascular cognitive impairment.

In this study, various neurological scores were assessed in patients with different levels of serum non-HDL-C, and patients with serum non-HDL-C levels higher than the normal value tended to present significant cognitive impairment and were accompanied by apparent mental behavior and emotion disorders, significantly affecting their ability and quality of life. Multivariable regression analyses found that the risk of cognitive disorders after acute ischemic stroke was increased significantly when non-HDL-C levels were higher than normal. Therefore, serum non-HDL-C levels were an independent risk factor of cognitive disorders after cerebral stroke. A recent study showed that high LDL-C levels and low HDL-C levels were associated with $\beta$-amyloid-associated cognitive impairment, suggesting that cholesterol levels play an important role in cognitive function [22]. Additional studies are necessary to assess their precise role in neurodegenerative diseases and cognitive impairment.

Damage from non-HDL-C to cognitive impairment after stroke could be associated with its strong association with the development of atherosclerosis. First, non-HDL-C particles include all potentially atherogenic lipoproteins [23-25]. Secondly, a high concentration of TG and VLDL-C in non-HDL-C reflects an increase of the liver's potential to generate lipoproteins causing atherosclerosis, leading to decreased interaction of these lipoproteins with liver receptors and reduced clearance. Therefore, these lipoproteins tend to be retained in blood circulation for a longer time, thereby promoting atherosclerosis. Finally, some lipoprotein remnants containing abundant TG may enter the arterial walls, leading to occurrence and development of atherosclerosis [7, 26]. Therefore, compared with LDL-C, serum non-HDL-C plays a higher effect in inducing atherosclerosis and LDL-C alone tend to neglect the promoting effect of other lipoproteins on ischemic stroke.

This study also showed that cognitive function scores were associated with the type of cerebral stroke in patients with high serum non-HDL-C levels and cerebral infarction. The score was the lowest in patients with $\mathrm{CACI} / \mathrm{PACI}$ and the highest in patients with LACI, indicating an association with injury to specific brain regions. CACI/PACI mainly involve the middle cerebral artery territory; a large lesion in the dominant hemisphere or bilateral hemispheric lesions can obviously involve the temporal lobe, insula, corpus callosum, hippocampus, and other parts that are closely associated with cognitive and memory functions, whereas the LACI normally has a smaller effect on the cognitive function unless the above specific locations are involved [27].

Cross-sectional analyses revealed that elevation of serum non-HDL-C levels were accompanied by elevated blood glucose and serum HCY levels. Previous studies found that patients with type 2 diabetes often suffered from dyslipidemia, especially patients with uncontrolled blood glucose. Abnormal lipid metabolism is mainly associated with diabetic vascular diseases, where specific lipid modulation or low-fat diet is likely to prevent or delay the progression of diabetic vascular diseases, suggesting that lipid metabolism disorders and diabetes have common pathophysiology for cognitive disorders after cerebral stroke [28]. In addition, patients with diabetes often also suffer from impaired renal function, and it has been shown that patients with decreased flomerular filtration rate also had greater levels of cognitive impairment [29]. Nevertheless, since the relationships of all these factors are complex, additional studies are necessary to assess comprehensively all these factors together.

In this study, regression analyses showed that the risk of cognitive disorders in patients with high serum $\mathrm{HCY}$ level was 1.057 times that of patients with a low level. which might be associated with high HCY-induced neurotoxicity [30]. These results agree with a recent study that showed that patients with vascular cognitive impairment have high HCY levels [31]. Accordingly, decreasing $\mathrm{HCY}$ levels seems to be associated with better cognitive outcomes in patients with Alzheimer's disease [32], suggesting that lowering HCY levels after an ischemic stroke could improve the outcomes, but additional studies are necessary to address this issue.

In addition, a low education level, age, high HAMD score, history of cerebral stroke, and family history of dementia were independent risk factors of cognitive disorders after ischemic stroke. These results are consistent with a previous study that showed that lower education, history of diabetes, high HAMD scores, high hsCRP levels, and high HbA1c levels were associated with 
cognitive impairment after acute ischemic stroke [33]. However, these results were all obtained in relatively small studies and need to be confirmed in larger trials.

This study is not without limitations. This was a single-center retrospective cohort study with a small sample size and limited follow-up duration. Only a limited number of variables could be assessed because of the retrospective nature of the study. Additional studies are necessary to confirm these findings.

\section{Conclusions}

In conclusion, high serum non-HDL-C levels might significantly increase the risk of cognitive disorders after acute cerebral stroke. As an inexpensive and easily measured biomarker, non-HDL-C screening could be undertaken for primary prevention of cognitive disorders after acute cerebral stroke in Chinese adults, but also provide a new direction for investigating treatment for cognitive disorders after acute cerebral stroke.

\section{Acknowledgments}

The authors acknowledge the invaluable participation of the patients.

\section{Funding}

This study was supported by the Scientific and Technical planing Project of Tianjin (13ZCZDSY01600) and Scientific and Technical Key Project of Tianjin Health Bureau (13KG121).

\section{Availability of data and materials}

The datasets analysed during the current study will not be publicly available to protect patient confidentiality.

\section{Authors' contributions}

$\mathrm{DL}$ and PL carried out the studies, participated in collecting data, and drafted the manuscript. YYZ and XLX performed the statistical analysis and participated in its design. HHZ, LPL and ZYT helped to draft the manuscript. All authors read and approved the final manuscript.

\section{Competing interests}

All authors declare that they have no competing interests.

\section{Consent for publication}

Not applicable.

\section{Ethics approval and consent to participate}

This study was conducted according to the Helsinki II Declaration and approved by the ethics committee at the Tianjin Huanhu Hospital. This study was written informed consent was obtained from every participant.

\section{Author details}

'Department of Neurology, Sixteenth wards, Tianjin Huanhu Hospital, Tianjin 300060, China. ${ }^{2}$ Department of Neurology, Second wards, Tianjin Huanhu Hospital, Tianjin 300060, China.

Received: 26 April 2016 Accepted: 10 August 2016

Published online: 26 August 2016

\section{References}

1. Sacco RL, Kasner SE, Broderick JP, Caplan LR, Connors JJ, Culebras A, et al. An updated definition of stroke for the 21st century: a statement for healthcare professionals from the American Heart Association/American Stroke Association. Stroke. 2013;44:2064-89.

2. Yew KS, Cheng E. Acute stroke diagnosis. Am Fam Physician. 2009;80:33-40.

3. Donnan GA, Fisher M, Macleod M, Davis SM. Stroke. Lancet. 2008;371:1612-23.
4. Cho SJ, Yu KH, Oh MS, Jung S, Lee JH, Koh IS, et al. Post-stroke memory impairment among patients with vascular mild cognitive impairment. BMC Neurol. 2014;14:244.

5. Zou Y, Zhu Q, Deng Y, Duan J, Pan L, Tu Q, et al. Vascular risk factors and mild cognitive impairment in the elderly population in Southwest China. Am J Alzheimers Dis Other Demen. 2014;29:242-7.

6. Expert Panel on Detection, Evaluation, and Treatment of High Blood Cholesterol in Adults. Executive summary of the third report of the national cholesterol education program (NCEP) expert panel on detection, evaluation, and treatment of high blood cholesterol in adults (adult treatment panel III). Jama. 2001;285:2486-97.

7. Jacobson TA, Ito MK, Maki KC, Orringer CE, Bays HE, Jones PH, et al. National lipid association recommendations for patient-centered management of dyslipidemia: part 1-full report. J Clin Lipidol. 2015;9:129-69.

8. Oremus M. Does the evidence say a 4-point change in ADAS-cog score is clinically significant? Alzheimers Dement. 2014;10:416-7.

9. Bamford J, Sandercock P, Dennis M, Burn J, Warlow C. Classification and natural history of clinically identifiable subtypes of cerebral infarction. Lancet. 1991;337:1521-6.

10. Molloy DW, Standish TI. A guide to the standardized Mini-Mental State Examination. Int Psychogeriatr. 1997;9 Suppl 1:87-94. discussion 143-50.

11. Hu JB, Zhou WH, Hu SH, Huang ML, Wei N, Qi HL, et al. Cross-cultural difference and validation of the Chinese version of Montreal Cognitive Assessment in older adults residing in Eastern China: preliminary findings. Arch Gerontol Geriatr. 2013;56:38-43.

12. Wang T, Xiao S, Li X, Wang H, Liu Y, Su N, et al. Reliability and validity of the Chinese version of the neuropsychiatric inventory in mainland China. Int J Geriatr Psychiatry. 2012;27:539-44.

13. Eto F, Tanaka M, Chishima M, Igarashi M, Mizoguchi T, Wada H, et al. Comprehensive activities of daily living (ADL) index for the elderly. Nihon Ronen Igakkai Zasshi. 1992;29:841-8.

14. Faries D, Herrera J, Rayamajhi J, DeBrota D, Demitrack M, Potter WZ. The responsiveness of the Hamilton Depression Rating Scale. J Psychiatr Res. 2000;34:3-10.

15. Cui Y, Blumenthal RS, Flaws JA, Whiteman MK, Langenberg P, Bachorik PS, et al. Non-high-density lipoprotein cholesterol level as a predictor of cardiovascular disease mortality. Arch Intern Med. 2001;161:1413-9.

16. Bittner V, Hardison R, Kelsey SF, Weiner BH, Jacobs AK, Sopko G. Non-highdensity lipoprotein cholesterol levels predict five-year outcome in the Bypass Angioplasty Revascularization Investigation (BARI). Circulation. 2002;106:2537-42.

17. Wu J, Chen S, Liu L, Gao X, Zhou Y, Wang C, et al. Non-high-density lipoprotein cholesterol vs low-density lipoprotein cholesterol as a risk factor for ischemic stroke: a result from the Kailuan study. Neurol Res. 2013;35:505-11.

18. Wu J, Chen S, Zhou Y, Wang C, Wang A, Zhang Q, et al. Non-high-density lipoprotein cholesterol on the risks of stroke: a result from the Kailuan study PLoS One. 2013;8:e74634

19. Wu J, Zhang Q, Yang H, Gao X, Zhou Y, Wang A, et al. Association between non-high-density-lipoprotein-cholesterol levels and the prevalence of asymptomatic intracranial arterial stenosis. PLoS One. 2013;8:e65229.

20. Zhu J, Wang Y, Li J, Deng J, Zhou H. Intracranial artery stenosis and progression from mild cognitive impairment to Alzheimer disease. Neurology. 2014;82:842-9.

21. Niu MJ, Yin FZ, Liu LX, Fang Y, Xuan XM, Wu GF. Non-high-density lipoprotein cholesterol and other risk factors of mild cognitive impairment among Chinese type 2 diabetic patients. J Diabetes Complications. 2013;27:443-6.

22. Reed B, Villeneuve S, Mack W, DeCarli C, Chui HC, Jagust W. Associations between serum cholesterol levels and cerebral amyloidosis. JAMA Neurol. 2014;71:195-200.

23. Berger JS, McGinn AP, Howard BV, Kuller L, Manson JE, Otvos J, et al. Lipid and lipoprotein biomarkers and the risk of ischemic stroke in postmenopausal women. Stroke. 2012:43:958-66.

24. Canoui-Poitrine F, LuC G, Bard JM, Ferrieres J, Yarnell J, Arveiler D, et al. Relative contribution of lipids and apolipoproteins to incident coronary heart disease and ischemic stroke: the PRIME Study. Cerebrovasc Dis. 2010:30:252-9.

25. Erqou S, Kaptoge S, Perry PL, Di Angelantonio E, Thompson A, White IR, et al. Lipoprotein(a) concentration and the risk of coronary heart disease, stroke, and nonvascular mortality. Jama. 2009;302:412-23. 
26. Jacobson TA, Ito MK, Maki KC, Orringer CE, Bays HE, Jones PH, et al. National Lipid Association recommendations for patient-centered management of dyslipidemia: part 1 - executive summary. J Clin Lipidol. 2014;8:473-88.

27. Sarangi S, San Pedro EC, Mountz JM. Anterior choroidal artery infarction presenting as a progressive cognitive deficit. Clin Nucl Med. 2000;25:187-90.

28. DeBoer MD. Obesity, systemic inflammation, and increased risk for cardiovascular disease and diabetes among adolescents: a need for screening tools to target interventions. Nutrition. 2013;29:379-86.

29. Kurella Tamura M, Xie D, Yaffe K, Cohen DL, Teal V, Kasner SE, et al. Vascular risk factors and cognitive impairment in chronic kidney disease: the Chronic Renal Insufficiency Cohort (CRIC) study. Clin J Am Soc Nephrol. 2011;6:248-56.

30. Ma H, Yan F, Li Z, Deng M, Qu Q. Investigation on plasma homocysteine level and cognition in population at high risk for stroke in Xi'an. Zhonghua Liu Xing Bing Xue Za Zhi. 2014;35:769-72.

31. Jiang $B$, Chen $Y$, Yao G, Yao C, Zhao H, Jia X, et al. Effects of differences in serum total homocysteine, folate, and vitamin B12 on cognitive impairment in stroke patients. BMC Neurol. 2014;14:217.

32. Cacciapuoti F. Lowering homocysteine levels with folic acid and B-vitamins do not reduce early atherosclerosis, but could interfere with cognitive decline and Alzheimer's disease. J Thromb Thrombolysis. 2013;36:258-62

33. Wang Y, Li P, Zhang M, Zhang H-h, Liu S, Zhou Y-y. Cross-sectional analysis of cognitive impairment and relative factors after acute ischemic stroke. Chi J Contemp Neurol Neurosurg. 2013;13:279-85.

\section{Submit your next manuscript to BioMed Central and we will help you at every step:}

- We accept pre-submission inquiries

- Our selector tool helps you to find the most relevant journal

- We provide round the clock customer support

- Convenient online submission

- Thorough peer review

- Inclusion in PubMed and all major indexing services

- Maximum visibility for your research

Submit your manuscript at www.biomedcentral.com/submit 